\title{
Research on the Art Image Query Method Based on Hierarchical Semantic and Incomplete Filtration
}

\author{
Cheng Mao ${ }^{1}$, Chai Wenlei ${ }^{2}$ and Wang Fuqiang * \\ ${ }^{1}$ No. 289 Lingyusi Street. Baoding, Hebei Province, P.R.C \\ ${ }^{2}$ No. 180 Wusidong Road. Baoding, Hebei Province, P.R.C \\ * No.402 Peace west road, Shijiazhuang, Hebei Province, P.R.C

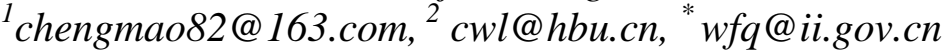

\begin{abstract}
Art image databases play an important role in the image research history. Art image can be classified into various categories, and each category has its own characteristics. Art image retrieval with high precision and speed can help the researchers understand an art image much more easily. In addition, the classification for the art images can help the researchers improve the working efficiency. In the art image retrieval, the query precision is much more important than the query speed. Improving the query precision at the cost of not seriously decreasing the query speed can be accepted. In this paper, a new method has been proposed to improve the query precision. The new method mainly includes initial query, reorganization, results recheck and images reordering. At the beginning, the tag query method and the semantic query method will be used to search the initial image results; then, the results will be reorganization according to the semantic method; finally, the images in the results will be filtered by the incomplete filtration method. According to the experimental results, the new method is proved that it can improve the query precision. The new method can be used in the art image retrieval process.
\end{abstract}

Keywords: art image, hierarchical semantic, retrieval, incomplete filtration, query precision

\section{Introduction}

So far, the art has considerable development and contains increasingly broad scope. It includes not only the ancient art [1-2], but also the modern art [3-4]. At the same time, it also includes both traditional art and electronic arts [5-6]. The form of the art is also different from before. Take the electronic art as an example, it also change to the network form in the developing process. Based on the development of Internet technology, the sharing of massive multimedia data such as image and video becomes a reality. At the same time, people are getting used to query information on the Internet. Under the current the Internet background, commercial search engines, such as Baidu, Bing, Google, etc., are committed to develop more intelligent image retrieval system. Traditional image retrieval method mainly relies on the text keywords input by the users, and the search engine will extend the keywords according to off-line built corpus to construct user query text feature. In the retrieval process, the image text feature will be constructed according to the webpage. The image retrieval results are obtained according to similarity calculation between characteristics of the user's query text features and image text [7-10].

Since the current Internet based on text retrieval technology [11-13] is already quite mature, text characteristics based image retrieval can be easily achieved. However, the problem lies on that the surrounding text labels and visual content of the images are not consistent. With the development of the image technology, search system with image directly as the query content is favored by many users. Current retrieval technology with image directly as the query content focuses on calculating the relevance scores of each 
piece of image according to the low-level visual features [14-16]. However, due to the low-level visual features of images can't well reflect the image semantic content, images with the same low-level visual features always can't prove the similar semantic content. In this way, search engine with both text method and image method can't be satisfactory for the direct search results.

In this context, the combination of image content to the initial retrieval results becomes a very important technology. With the development of image retrieval technology, a lot of image re ranking methods have been proposed. The classical methods, including clustering, classification, etc., transform the image ranking problem into a classical semi supervised learning problem to realize the filtering of the image. The method based on machine learning can automatically learn the ranking function favored by the user according to the initial search results order, and improve the final image retrieval ranking. Combined with the current image re ranking technology [17], the performance of the current image retrieval has been greatly improved.

However, traditional image re ranking techniques often do not consider the semantic etcontent of the image information. The problem of the semantic gap of the image is still not resolved. The research trend is how to combine the semantic content information of the image to improve higher ranking performance and avoid the image semantic gap [1819].

At the same time, retrieval of art images has its own characteristics, and most users of the art images are professionals. Retrieval of art images is generally used for the analysis and identification of images, classification and sorting of images, and so on, which lead to a certain difference between the artistic images and ordinary image recognition. In order to properly retrieve the art images, this paper develops a new method. The main work of this paper is to establish a new image retrieval method, which can help to improve the performance of the art image query. The remainder of the paper is shown as the following: some basic query methods are introduced in Section 2; the new method is described in Section 3; the verification is shown in Section 4; and the Conclusion is shown in Section 5 .

\section{The Basic Query Methods}

So far, image reordering method is mainly according to the image visual features. The image reordering is realized by mining query image correlation information. The main features include edge histogram, bow histogram, color moments and so on. These methods can be classified into several categories:

(1) Clustering based image re ranking

This kind of method introduces the assumption conditions. It means that the initial retrieval reordering results and the query related images are similar to each other and the major images in the query results are similar. Through the clustering method, similar images can be clustered into dense images clusters, and the not similar images will be clustered into relatively sparse clusters. Then, these images in a sparse image cluster will be put back, while images in the dense cluster will be put to the front position. The retrieval ranking performance will be improved. This thought is the core of cluster re ranking based methods. The key to this kind of method is how to put the similar images together into a class. In fact, for the clustering problem, a kind of method of information bottleneck based method has been proposed. This method can be used to obtain the mutual information entropy of each class according to initial relevance scores and the initial cluster of each piece image to be retrieved to dynamically adjust the image contained in each class. Finally, the images are reordered according to consider the correlation between each image, correlation degree in corresponding relationship between classes belonged and denseness of the corresponding class. The method is mainly used in the condition of image retrieval results with high visual repetition rate. It can get better 
image reordering results, but the effective limitation is often fit for the initial retrieval ranking results with a single visual mode. When the return type of the image is much more and big visual difference exists between the returned images, this kind of method can't improve the quality of the retrieval results. On the other hand, because the number of clustering is the key parameters of this kind of method, how to dynamic adaptively determine the number of clusters is still a difficult problem in the current research, which also limits this method in actual application from a certain extent.

(2) Classification based image re ranking [22]

There are two kinds of images in the results of image retrieval: similar and not similar. The image classification problem can be regarded as an image classification problem. Classification based method often needs the initial search ranking results to estimate the pseudo correlations and obtain the corresponding pseudo positive instances and pseudo negative instances. These pseudo positive instances and negative pseudo instances will be used to train the classifier for the estimation of correlation of the retrieval results. The trained classifier is used to predict the sort images, and reordering the images through the predicted scores. The key to this method is how to choose the non-pollution training sample and how to get the classifier with high accuracy. Here, a typical method is given to introduce the method. Pseudo relevance feedback method is referred to reorder the images. According to an initial search results ranking, the top $k$ images will be done as positive examples. At the same time, relevant images for query only account a small proportion in the large-scale database, therefore, $\mathrm{k}$ images selected randomly in the database can be used as the negative examples in order to acquire the training samples. For the training of the classifier, the main methods are boosting, SVM, and SVM Ranking, etc. More importantly, for the retrieval activity, the problem of time should be considered. On the other hand, in order to estimate the parameters of the classification model accurately, it is necessary to have a pure training sample with sufficient number. However, in this method, the number of training samples obtained is often limited and with noisy images, which limit the application of this method in practices.

(3) Image re ranking based on graph structure [23-24]

The graph structure based re ranking method can be regarded each image as a node, and the similarity between images can be regarded as the weight of the connection edge between the nodes. Then, re ranking work can be realized by mutual transfer between edges in the image according to the correlation fraction. This method focuses on how to construct the reasonable structure to make the connection between the real images of significant and connection weights between different images small. Based on the image structure, relevance scores estimation problem for the image can be seen as a random walk process in the figure. The ranking score of each image can be calculated by the stable probability of random walk of each image node. The famous Page Rank algorithm is also can be used in the image retrieval field. The art images to be sorted are regarded as the text, and the image visual similarity is regarded as the hyperlinks between images. Based on the two conditions, classical Page Rank algorithm is used to calculate the importance of per image node. From the perspective of semi supervised learning and the analysis of the constructed image model, the online learning scheduling model can be learned according to the image correlation label transmission. In this kind of method, the key lies in how to reasonably construct the connected image model between the images nodes. It is similar with the cluster methods, for the conditions of diverse visual patterns in the initial retrieval results, structure based image re ranking approach is often unable to obtain good effect.

(4) Learning based image re ranking method [25]

Image re ranking method based on the learning theory is supported by modern machine learning theory. The image ranking problem is considered as the traditional machine learning problem. According to the initial image retrieval results, this kind of method can be divided into some steps: 1) mining a prior information; 2) training the prediction 
function for the image correlation; 3) make a prediction for each image according to the prediction function; 4) using the prediction scores as relevance scores of each piece image to be the ranking; 5) obtaining the final ranking results. The key to this method is how to search for prior information which can reflect the relevance of the image according to the initial search results. From the perspective of image historical click rate, image with high historical click rate may often be the images which attract the attention of the users. Combined with Gaussian process regression model, the prediction function aiming at the image click rate is trained, and finally, prediction scores and the initial image ranking score are combined to realize the re ranking process of the images to be sorted in the initial retrieval results. From the perspective of image multi feature fusion, the query method can be divided into the following steps: 1) semantic model relative to the user queries is analyzed; 2) search the query relevant point of reference image; 3) fusion coefficients of each feature channel should be adaptive learned based on the principle that multiple feature channels corresponding to retrieval ordering structure should be similar; 4) the ranking of the final results is guided by the distance measure of the fusion image. The advantage of the method based on learning is that the adaptive ranking function can be obtained by combining the features of the user query itself, but the training time is often relatively long.

In order to develop a suitable method which can give consideration to both query precision and query speed, the advantages of each method can be combined to form a new method for the art image retrieval.

\section{A New Art Image Query Method}

\subsection{Hierarchical Semantic Retrieval}

If $\left(s_{1, j}, s_{2, j}, \ldots, s_{h, j}\right)$ is used to describe distribution characteristics of each semantic concept on the image layer $i$, and $\left(w_{j, 1}, w_{j, 2}, \ldots, w_{j, m}\right)$ is used to describe relevance weights of semantic concept $j$ on the next layer and each semantic concept on the image layer $i$, the calculation method of membership score of semantic concept $j$ on the corresponding image layer $i+1$ can be expressed as the following figure:

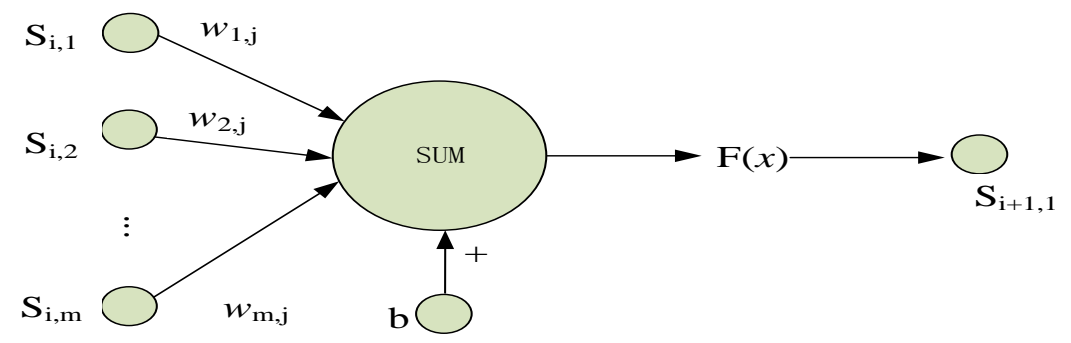

Figure 1. Semantic Hierarchy Connection Diagram

In the Figure.1, $F(x)$ is the sigmoid function. If need to express the image with semantic hierarchy layer, two main contents of the semantic concept and the connection weight $\left(w_{j, 1}, w_{j, 2}, \ldots, w_{j, m}\right)$ should be known.

The semantic concept will be obtained by the cluster method. If $T_{i}=\left\{s_{j, i}\right\}, j=1,2, \ldots, n$ is used to describe the distribution of the training image sets on the layer $i$ and $C_{i+1}=\left\{c_{r, j+1}\right\}, r=1,2, \ldots, h$ is the set of semantic concept on layer $i+1$. Then the semantic concept can be obtained by the following method: 
(1) Randomly select $k$ samples as the cluster center and it can be described as $\mu_{1}, \ldots, \mu_{k}$;

(2) For each image $t$ in the training set, it should be calculate its ownership according to the following equation:

$$
c_{m}: \underset{m}{\arg \min }\left\|S_{t, i}-\mu_{m}\right\|^{2}
$$

(3) For each class, update the centroid:

$$
\mu j=\frac{\sum_{l=1}^{p} s_{l, i}}{p}, \quad s_{l, i} \in c_{m}
$$

(4) If the difference between the two iteration clustering centers is less than a certain threshold, the algorithm converges and the iteration stops.

(5) Output the semantic concept $C_{i+1}=\left\{c_{r, j+1}\right\}=\left\{c_{r, j}\right\}, m=1, \ldots, h$. Here, the semantic concept is obtained.

For the connection weight $\left(w_{j, 1}, w_{j, 2}, \ldots, w_{j, m}\right)$, it will be obtained from the hyper plane. The plane can be expressed as:

$$
w^{T} x+b=0
$$

Then, the classification function can be expressed as:

$$
g(x)=w^{T} x+b
$$

Function value of function classification is used to represent the membership scores of the semantic concept of the image, and the purpose of learning is to determine the two parameter $w$ and $b$ in the classification function $g(x)$ according to a set of training images. If defining two extreme planes in parallel, the corresponding optimization objective function can be written as:

$$
\begin{aligned}
& \min \frac{1}{2}\|w\|^{2} \\
& \text { s.t. } y_{i}\left(w^{T} x_{i}+b\right) \geq 1, i=1, \ldots, n
\end{aligned}
$$

Then, the connection weight $\left(w_{j, 1}, w_{j, 2}, \ldots, w_{j, m}\right)$ is known.

\subsection{Image Incomplete Filtration}

Incomplete filtration is used to obtain the query results according to divide the inverted list into some sublist and clustering centers methods. The process is accomplished by training clustering centers when constructing an inverted index.

For the query image feature $q$, combined with the incomplete filtration method, each detailed step can be described as the following:

Step1: Calculates the distance $D_{1}=\left\{d_{1}, d_{2}, \ldots, d_{k 1}\right\}$ of $q$ to all clustering centers $C_{1}=\left\{d_{1}, d_{2}, \ldots, d_{k 1}\right\}$.

Step2: The smallest $w$ values in $D_{1}$ should be found, and the correspond cluster centers will be $C_{c q}=\left\{c_{q, 1}, c_{q, 2}, \ldots, c_{q, w}\right\}$;

Step3: Merge the $w$ cluster centers and the corresponding the second level clustering centers into the set $C_{c q}=\bigcup_{i=1}^{w} C_{q, i}=\bigcup_{i=1}^{w} \bigcup_{j=1}^{k 2} c_{q, i j}$

Step4: Construct a hypersphere $S_{q}$, the corresponding radius $R_{q}$ can be obtained by $q$ and minimum distance of $w$ cluster centers. 
Step5: Filteration not similar results. Calculate the distance of query character $q$ and all the Euclidean distance among all the clustering centers in $C_{c q^{\prime}}$. According to the $d\left(q, c_{c q, i_{j}}\right)=\left\|x-c_{c q, i_{j}}\right\| \leq R_{q},\left(i=1, \ldots, w, j=1, \ldots, k_{2}\right)$, the results set will be obtained for reordering.

Step6: Non symmetric distance method in residuals based quantized retrieval method will be used in the reordering process according to the distance between query feature $q$ and features in $R S_{q}$, where $R S_{q}=\left\{y_{1}, \ldots, y_{m}\right\}$.

\subsection{The New Method}

The new method is composed of the steps shown above, and it mainly includes initial query, reorganization, results recheck and images reordering. At the beginning, the tag query method and the semantic query method will be used to search the initial image results; then, the results will be reorganization according to the semantic method; finally, the images in the results will be filtered by the incomplete filtration method. The flow chart of the new query method can be shown in Figure. 2

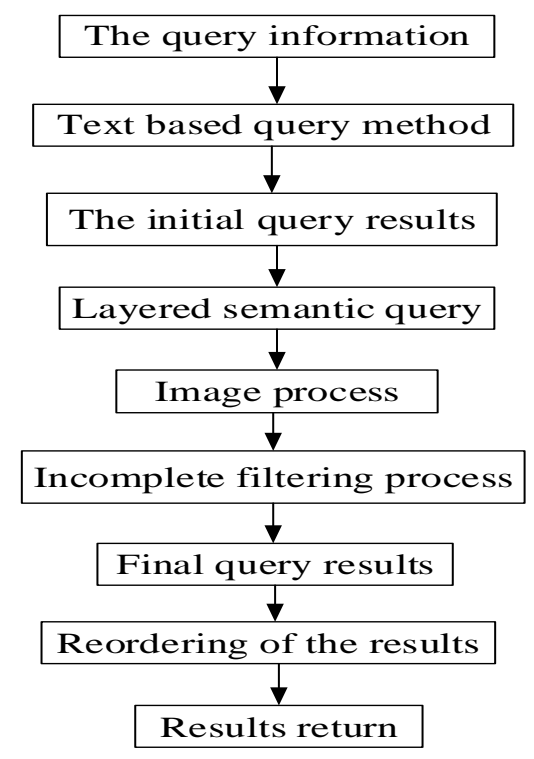

\section{Figure 2. Flow Chart of the New Query Method}

\section{Verification}

In order to predict the retrieval performance of the new proposed method, a series of experiments have been designed. According to comparison of the query precision and query speed, the performance of the new proposed method will be tested. Due to the aim of the experiments used for the verification of precision and speed, the number of feature points won't be given in the results. The dataset are used in reference [26] and reference [27] and the retrieval results of the new proposed method are used to compare with that referred to reference [27]. Specific information of the datasets is given in Table 1. All the experiments are worked out by software of mat lab R2011b on a computer with Intel Core I5 with the frequency of $2.8 \mathrm{GHz}$ and $4 \mathrm{G}$ memory. 
Table 1. Dataset Used for the Experiment

\begin{tabular}{|c|c|c|}
\hline Dataset & sift & gist \\
\hline Feature dimension & 128 & 960 \\
\hline Training set & 100000 & 500000 \\
\hline Dataset & 1000000 & 1000000 \\
\hline Query set & 10000 & 1000 \\
\hline
\end{tabular}

The Table. 2 to Table. 4 gives the results generated by the new method and the preferred method. Parameters of $\mathrm{k}$ and $\mathrm{w}$ are the inverted list number of the index structure and the number of inverted permutation table. The parameter of $\mathrm{k}$ is 64, 256 and 1024 and parameter of $\mathrm{w}$ is 8,16 , and 32 for dataset sift, while parameter of $\mathrm{k}$ is 64 and 256 and parameter of $w$ is 8 and 16 for dataset gist. In the tables, $R$ is the query results with the number of 100 .

The Table. 2 gives the query precision and query time of the new method and the reference method. The query time can reflect the query speed of the method. From the table, we can see that the query precision of the new method is a little higher than that of a reference query method, and the query time is increased.

\section{Table 2. The Retrieval Performance of the Reference and New Methods}

\begin{tabular}{|c|c|c|c|c|}
\hline \multirow{2}{*}{ Sift } & \multicolumn{2}{|c|}{ Recall @ 100 } & \multicolumn{2}{c|}{ Query time(ms) } \\
\cline { 2 - 5 } & $\begin{array}{c}\text { Reference } \\
\text { method }\end{array}$ & $\begin{array}{c}\text { The new } \\
\text { method }\end{array}$ & $\begin{array}{c}\text { Reference } \\
\text { method }\end{array}$ & $\begin{array}{c}\text { The new } \\
\text { method }\end{array}$ \\
\hline $\mathrm{k}=64 / \mathrm{w}=8$ & 0.94 & 0.95 & 21.8 & 30.2 \\
\hline $\mathrm{k}=256 / \mathrm{w}=16$ & 0.94 & 0.948 & 11.8 & 19.7 \\
\hline $\mathrm{k}=1024 / \mathrm{w}=32$ & 0.95 & 0.961 & 6.9 & 11.4 \\
\hline gist & $/$ & $/$ & $/$ & $/$ \\
\hline $\mathrm{k}=64 / \mathrm{w}=8$ & 0.66 & 0.68 & 27.1 & 36.2 \\
\hline $\mathrm{k}=256 / \mathrm{w}=16$ & 0.66 & 0.677 & 15.9 & 21.7 \\
\hline
\end{tabular}

In order to get the detailed performance of the new method, the query precision and query time of the new method with different parameters have been calculated. Query performance of the new method is verified according to the variation of parameters of $\mathrm{k} 2$ and $\lambda$.

From the Table. 3 and Table.4, it can be seen that the new method improve the query precision to some extent. This is due to the semantic retrieval before the incomplete filtration. If want to improve the query precision, the first step is that the initial image results include all the relative images without missing. In the new method, the text query and semantic query provide all the relative images in the initial results. Then, the incomplete filtering method is used to realize the aim of the images ranking. The most relative images will be put in front of the results list. Meanwhile, the query time becomes longer, and this is due to the addition of the semantic query step. When the query time is long, the total query time has little difference. However, it may be paid lots of attention that the query time will be increased a lot when the query time is short. Therefore, the next step to study is how to reduce the search time. 
Table 3. Query Performance of the Two Methods in Dataset Sift with Different $\lambda$ and $K_{2}$

\begin{tabular}{|c|c|c|c|c|c|c|}
\hline \multirow{2}{*}{ Sift } & $k_{2}$ & $\lambda$ & \multicolumn{2}{c|}{ Recall@ 100 } & \multicolumn{2}{c|}{ Query time } \\
\cline { 4 - 7 } & & & $\begin{array}{c}\text { Reference } \\
\text { method }\end{array}$ & $\begin{array}{c}\text { The new } \\
\text { method }\end{array}$ & $\begin{array}{c}\text { Reference } \\
\text { method }\end{array}$ & $\begin{array}{c}\text { The new } \\
\text { method }\end{array}$ \\
\hline $\mathrm{k}=64 / \mathrm{w}=8$ & 4 & 0.97 & 0.94 & 0.951 & 10.8 & 14.7 \\
\hline $\mathrm{k}=64 / \mathrm{w}=8$ & 4 & 0.98 & 0.94 & 0.948 & 10.4 & 14.3 \\
\hline $\mathrm{k}=64 / \mathrm{w}=8$ & 4 & 0.99 & 0.93 & 0.937 & 9.6 & 13.1 \\
\hline $\mathrm{k}=64 / \mathrm{w}=8$ & 16 & 0.97 & 0.94 & 0.949 & 8.1 & 11.9 \\
\hline $\mathrm{k}=64 / \mathrm{w}=8$ & 16 & 0.98 & 0.94 & 0.951 & 7.6 & 10.2 \\
\hline $\mathrm{k}=64 / \mathrm{w}=8$ & 16 & 0.99 & 0.93 & 0.94 & 7.0 & 9.8 \\
\hline $\mathrm{k}=64 / \mathrm{w}=8$ & 48 & 0.97 & 0.84 & 0.852 & 7.4 & 9.9 \\
\hline $\mathrm{k}=64 / \mathrm{w}=8$ & 48 & 0.98 & 0.94 & 0.951 & 6.9 & 9.4 \\
\hline $\mathrm{k}=64 / \mathrm{w}=8$ & 48 & 0.99 & 0.93 & 0.943 & 6.5 & 9.1 \\
\hline $\mathrm{k}=256 / \mathrm{w}=16$ & 4 & 0.94 & 0.94 & 0.955 & 7.1 & 10.2 \\
\hline $\mathrm{k}=256 / \mathrm{w}=16$ & 4 & 0.95 & 0.94 & 0.953 & 6.6 & 9.5 \\
\hline $\mathrm{k}=256 / \mathrm{w}=16$ & 4 & 0.96 & 0.93 & 0.938 & 6.0 & 9.2 \\
\hline $\mathrm{k}=256 / \mathrm{w}=16$ & 16 & 0.94 & 0.94 & 0.947 & 5.3 & 8.2 \\
\hline $\mathrm{k}=256 / \mathrm{w}=16$ & 16 & 0.95 & 0.94 & 0.95 & 5.7 & 8.5 \\
\hline $\mathrm{k}=256 / \mathrm{w}=16$ & 16 & 0.96 & 0.93 & 0.946 & 5.0 & 7.0 \\
\hline $\mathrm{k}=256 / \mathrm{w}=16$ & 48 & 0.94 & 0.94 & 0.943 & 5.1 & 7.1 \\
\hline $\mathrm{k}=256 / \mathrm{w}=16$ & 48 & 0.95 & 0.94 & 0.946 & 4.9 & 7.2 \\
\hline $\mathrm{k}=256 / \mathrm{w}=16$ & 48 & 0.96 & 0.93 & 0.938 & 4.6 & 7.1 \\
\hline $\mathrm{k}=1024 / \mathrm{w}=32$ & 4 & 0.95 & 0.95 & 0.957 & 4.5 & 6.9 \\
\hline $\mathrm{k}=1024 / \mathrm{w}=32$ & 4 & 0.96 & 0.95 & 0.955 & 4.2 & 6.6 \\
\hline $\mathrm{k}=1024 / \mathrm{w}=32$ & 4 & 0.97 & 0.94 & 0.946 & 4.0 & 6.2 \\
\hline $\mathrm{k}=1024 / \mathrm{w}=32$ & 16 & 0.95 & 0.95 & 0.954 & 4.0 & 6.3 \\
\hline $\mathrm{k}=1024 / \mathrm{w}=32$ & 16 & 0.96 & 0.95 & 0.956 & 3.9 & 6.1 \\
\hline $\mathrm{k}=1024 / \mathrm{w}=32$ & 16 & 0.97 & 0.94 & 0.948 & 3.7 & 6.1 \\
\hline $\mathrm{k}=1024 / \mathrm{w}=32$ & 48 & 0.92 & 0.95 & 0.956 & 4.8 & 7.4 \\
\hline $\mathrm{k}=1024 / \mathrm{w}=32$ & 48 & 0.93 & 0.95 & 0.957 & 4.4 & 7.2 \\
\hline $\mathrm{k}=1024 / \mathrm{w}=32$ & 48 & 0.94 & 0.94 & 0.948 & 4.2 & 7.2 \\
\hline & & & & & & \\
\hline
\end{tabular}

Table 4. Table 3 Query Performance of the Two Methods in Dataset Gift with Different $\lambda$ and $K_{2}$

\begin{tabular}{|c|c|c|c|c|c|c|}
\hline \multirow{2}{*}{ Gist } & $k_{2}$ & $\lambda$ & \multicolumn{2}{c|}{ Recall @ 100 } & \multicolumn{2}{c|}{ Query time } \\
\cline { 4 - 7 } & & & $\begin{array}{c}\text { Reference } \\
\text { method }\end{array}$ & $\begin{array}{c}\text { The new } \\
\text { method }\end{array}$ & $\begin{array}{c}\text { Reference } \\
\text { method }\end{array}$ & $\begin{array}{c}\text { The new } \\
\text { method }\end{array}$ \\
\hline $\mathrm{k}=64 / \mathrm{w}=8$ & 4 & 0.98 & 0.66 & 0.665 & 16.2 & 21.2 \\
\hline $\mathrm{k}=64 / \mathrm{w}=8$ & 4 & 0.99 & 0.66 & 0.667 & 14.3 & 18.3 \\
\hline $\mathrm{k}=64 / \mathrm{w}=8$ & 4 & 1 & 0.65 & 0.658 & 11.4 & 14.2 \\
\hline $\mathrm{k}=64 / \mathrm{w}=8$ & 16 & 0.98 & 0.66 & 0.666 & 13.4 & 17.5 \\
\hline $\mathrm{k}=64 / \mathrm{w}=8$ & 16 & 0.99 & 0.66 & 0.665 & 12.1 & 16.8 \\
\hline $\mathrm{k}=64 / \mathrm{w}=8$ & 16 & 1 & 0.65 & 0.654 & 9.5 & 13.2 \\
\hline $\mathrm{k}=64 / \mathrm{w}=8$ & 48 & 0.98 & 0.66 & 0.665 & 12.8 & 15.4 \\
\hline $\mathrm{k}=64 / \mathrm{w}=8$ & 48 & 0.99 & 0.66 & 0.667 & 10.7 & 14.2 \\
\hline $\mathrm{k}=64 / \mathrm{w}=8$ & 48 & 1 & 0.65 & 0.658 & 9.1 & 13.2 \\
\hline $\mathrm{k}=256 / \mathrm{w}=16$ & 4 & 0.97 & 0.67 & 0.675 & 13.0 & 16.8 \\
\hline $\mathrm{k}=256 / \mathrm{w}=16$ & 4 & 0.98 & 0.67 & 0.677 & 10.2 & 14.4 \\
\hline
\end{tabular}




\begin{tabular}{|c|c|c|c|c|c|c|}
\hline $\mathrm{k}=256 / \mathrm{w}=16$ & 4 & 0.99 & 0.66 & 0.67 & 8.7 & 12.0 \\
\hline $\mathrm{k}=256 / \mathrm{w}=16$ & 16 & 0.98 & 0.67 & 0.675 & 9.4 & 13.2 \\
\hline $\mathrm{k}=256 / \mathrm{w}=16$ & 16 & 0.99 & 0.67 & 0.675 & 8.5 & 11.8 \\
\hline $\mathrm{k}=256 / \mathrm{w}=16$ & 16 & 1 & 0.66 & 0.667 & 6.7 & 9.4 \\
\hline $\mathrm{k}=256 / \mathrm{w}=16$ & 48 & 0.99 & 0.67 & 0.677 & 8.7 & 12.1 \\
\hline $\mathrm{k}=256 / \mathrm{w}=16$ & 48 & 1 & 0.67 & 0.676 & 7.7 & 11.4 \\
\hline $\mathrm{k}=256 / \mathrm{w}=16$ & 48 & 1.1 & 0.22 & 0.32 & 4.3 & 7.3 \\
\hline
\end{tabular}

\section{Conclusion}

Art image retrieval with high precision and speed can help the researchers understand an art image much more easily, and the classification for the art images can help to improve the working efficiency. In the art image retrieval, the query precision is much more important. The art image retrieval method is similar to the image retrieval. There are also various methods for the image query. No matter text based or visual features based method can't be suitable for the content based image retrieval, so it should develop a new method for the art image query.

In this paper, a new method has been proposed to improve the query precision. The new method mainly includes initial query, reorganization, results recheck and images reordering. At the beginning, the tag query method and the semantic query method will be used to search the initial image results; then, the results will be reorganization according to the semantic method; finally, the images in the results will be filtered by the incomplete filtration method.

According to the experimental results, the new method is proved that it can improve the query precision to some extent. This is due to the semantic retrieval before the incomplete filtration. The initial image results should include all the relative images without missing to keep the quality of the initial query results. In the new method, the text query and semantic query provide all the relative images in the initial results. Then, the incomplete filtering method is used to realize the aim of the images ranking. However, the new method increases the query time due to the addition of the semantic query step. Therefore, the next step to study is how to reduce the search time. The new method can be used in the art image retrieval process.

\section{References}

[1] H. Y. Rao, B. Li, Y. M. Yang, Q. L. Ma and C. S. Wang, "Proteomic identification of organic additives in the mortars of ancient Chinese wooden buildings", Analytical Methods, vol. 2015, no. 1, (2015), pp. 143-149.

[2] M. Manfredi, E. Barberis, A. Rava, E. Robotti, F. Gosetti and E. Marengo, "Portable diffuse reflectance infrared Fourier transform (DRIFT) technique for the non-invasive identification of canvas ground: IR spectra reference collection", Analytical Methods, vol. 2015, no. 6, (2015), pp. 2313-2322.

[3] L. Caforio, M. E. Fedi, P. A. Mando, F. Minarelli, E. Peccenini, V. Pellicori, F. C. Petrucci, P. Schwartzbaum and F. Taccetti, "Discovering forgeries of modern art by the C-14 Bomb Peak", European Physical Journal Plus, vol. 2014, no. 1, (2014).

[4] M. Krischel, "A Stone Never Cut For: Cranial Lithotomy in Early Modern Art", Journal of Urology, vol. 2013, no. 4, (2013), pp. E449-E450.

[5] R. K. Cady and K. Farmer, "Acupuncture in the Treatment of Headache: A Traditional Explanation of an Ancient Art”, Headache, vol. 2015, no. 3, (2015), pp. 457-464.

[6] M. R. Guasp, J. A. A. Daviu and G. A. Capolino, "Advances in Electrical Machine, Power Electronic, and Drive Condition Monitoring and Fault Detection: State of the Art", IEEE Transactions on Industrial Electronics, vol. 2015, no. 3, (2015), pp. 1746-1759.

[7] S. F. Lin, C. C. Wu, C. Y. Hsu and D. C. Hsu, "An Efficient 3d Model Retrieval Based on Principal Axes Analysis and Feature Integration", International Journal of Pattern Recognition and Artificial Intelligence, vol. 2011, no. 4, (2011), pp. 583-604.

[8] R. Zhu and M. Yao, "Image feature optimization based on nonlinear dimensionality reduction", Journal of Zhejiang University-Science A, vol. 2009, no. 12, (2009), pp. 1720-1737. 
[9] S. M. Hsieh and C. C. Hsu, "Retrieval of images by spatial and object similarities", Information Processing \& Management, vol. 2008, no. 3, (2008), pp. 1214-1233.

[10] S. Dominich, J. Goth, T. Kiezer and Z. Szlavik, "An entropy-based interpretation of retrieval status value-based retrieval, and its application to the computation of term and query discrimination value", Journal of the American Society for Information Science and Technology, vol. 2004, no. 7, (2004), pp. 613-627.

[11] F. Wu, X. Y. Jiang, X. Li, S. L. Tang, W. M. Lu, Z. F. Zhang and Y. T. Zhuang, "Cross-Modal Learning to Rank via Latent Joint Representation", IEEE Transactions on Image Processing, vol. 2015, no. 5, (2015), pp. 1497-1509.

[12] M. U. Kim and K. Yoon, "Performance evaluation of large-scale object recognition system using bagof-visual words model", Multimedia Tools and Applications, vol. 2015, no. 7, (2015), pp. 2499-2517.

[13] H. Wu and L. L. He, "Combining visual and textual features for medical image modality classification with 1(p)-norm multiple kernel learning", Neurocomputing, vol. 2015, (2015), pp. 387-394.

[14] H. C. Huang, D. Y. Wang, L. Rong, X. Zhou, Z. Y. Li and Y. X. Wang, "Application of autofocusing methods in continuous-wave terahertz in-line digital holography”, Optics Communications, vol. 2015, (2015), pp. 93-98

[15] J. Yu, C. Q. Hong, D. P. Tao and M. Wang, "Semantic embedding for indoor scene recognition by weighted hypergraph learning", Signal Processing, vol. 2015, (2015), pp. 129-136.

[16] Y. Zhang, T. Guan, L. Y. Duan, B. C. Wei, J. Gao and T. Mao, "Inertial sensors supported visual descriptors encoding and geometric verification for mobile visual location recognition applications", Signal Processing, vol. 2015, (2015), pp. 17-26.

[17] H. Y. Lee and L. S. Lee, "Enhanced Spoken Term Detection Using Support Vector Machines and Weighted Pseudo Examples", IEEE Transactions on Audio Speech and Language Processing, vol. 2013, no. 6, (2015), pp. 1272-1284

[18] S. D. Roy, K. Bhardwaj, R. Garg and S. Chaudhury, "Camera-based document image matching using multi-feature probabilistic information fusion", Pattern Recognition Letters, vol. 2015, (2015), pp. 42-50.

[19] F. X. Feng, R. F. Li and X. J. Wang, "Deep correspondence restricted Boltzmann machine for crossmodal retrieval", Neurocomputing, vol. 2015, (2015), pp. 50-60.

[20] A. Alaybeyoglu, "Transmission of image data using fuzzy logic based clustering infrastructure in mobile multimedia sensor networks", Journal of Intelligent \& Fuzzy Systems, vol. 2015, no. 3, (2015), pp. 1235-1242.

[21] S. Kala and S. Vasuki, "Feature correlation based parallel hyper spectral image compression using a hybridization of FCM and subtractive clustering", Journal of Communications Technology and Electronics, vol. 2014, no. 12, (2014), pp. 1378-1389.

[22] M. B. Zhao, C. J. Zhan, Z. Wu and P. Tang, "Semi-Supervised Image Classification Based on Local and Global Regression”, IEEE Signal Processing Letters, vol. 2015, no. 10, (2015), pp. 1666-1670.

[23] D. C. G. Pedronette, O. A. B. Penatti and R. D. Torres, "Unsupervised manifold learning using Reciprocal kNN Graphs in image re-ranking and rank aggregation tasks", Image and Vision Computing, vol. 2014, no. 2, (2014), pp. 120-130.

[24] D. C. G. Pedronette and R. D. Torres, "Exploiting pairwise recommendation and clustering strategies for image re-ranking", Information Sciences, vol. 2012, (2012), pp. 19-34.

[25] M. Xi, W. T. Lu, J. Yang, Y. Ma, W. Yao and Z. C. Zheng, "A hybrid method based on extreme learning machine and k-nearest neighbor for cloud classification of ground-based visible cloud image", Neurocomputing, vol. 2015, (2015), pp. 238-249.

[26] J. Herve, D. Matthijs and S. Cordelia, "Product quantization for nearest neighbor search", IEEE Transactions on Pattern Analysis and Machine Intelligence, vol. 33, no. 1, (2011), pp. 117-128.

[27] L. F. Ai, J. Q. Yu, T. Guan and Y. F. He, "Adaptively Filtering Query Results for Large Scale Image Feature Retrieval”, Chinese Journal of Computers, vol. 37, no. 30, (2014), pp. 1-12. 